\title{
Physicochemical Properties of Diosmetin and Lecithin Complex
}

\author{
Korbanjhon Brad ${ }^{1 *}$ and Chungang Chen ${ }^{2}$ \\ ${ }^{1}$ College of Chemical and Biological Sciences, Yili Normal University, Yining 835000, Xinjiang, ${ }^{2}$ School of Food Science, Henan \\ Institute of Science and Technology, Xinxiang 453003, China
}

*For correspondence: Email: korbanjhon_yili@126.com

Received: 21 April 2012

Revised accepted: 7 July 2013

\begin{abstract}
Purpose: To improve the hydrophobicity of diosmetin, the complex of diosmetin and lecithin was prepared and its physicochemical properties were characterized in this study.

Methods: The complex of diosmetin and lecithin was prepared by solvent method. The physicochemical properties of the complex were investigated by ultraviolet-visible spectrometry (UV/Vis), infrared spectrometry (IR), scanning electron microscopy (SEM), differential scanning calorimetry (DSC) and x-ray diffractometry (XRD).

Results: Based on UV and IR analysis, there was no significant difference between the physical mixture and the complex while SEM showed that diosmetin in the complex did not present a crystal appearance but rather was dispersed in lecithin. DSC thermograms of the complex mainly showed that the presence of lecithin caused the characteristic endothermal peaks of diosmetin to disappear while $x$-diffractograms showed that the crystalline peaks of diosmetin was absent in the complex.

Conclusion: The complex is held together by non-covalent-bond, and thus a new compound was not formed. Diosmetin in the complex is molecularly dispersed in the lecithin matrix.
\end{abstract}

Keywords: Diosmetin, Lecithin, Complex, Physicochemical, Non-covalent bond

Tropical Journal of Pharmaceutical Research is indexed by Science Citation Index (SciSearch), Scopus, International Pharmaceutical Abstract, Chemical Abstracts, Embase, Index Copernicus, EBSCO, African Index Medicus, JournalSeek, Journal Citation Reports/Science Edition, Directory of Open Access Journals (DOAJ), African Journal Online, Bioline International, Open-J-Gate and Pharmacy Abstracts

\section{INTRODUCTION}

Diosmetin (3',5,7-trihydroxy-4'-methoxyflavone) is a bioflavonoid found in spearmint, oregano, and many other plants. Like all bioflavonoids, diosmetin helps maintain normal capillary permeability and fragility [1-3]. But for its poor solubility in oil, the application of diosmetin in functional food and medicine is restricted. The low hydrophobicity of diosmetin could result in its poor permeation across the intestinal epithelial cells and reduce the gastrointestinal tract absorption, which could decrease its oral bioavailability. Lecithin is a group of yellowbrownish fatty substances occurring in animal and plant tissues, and in egg yolk [4]. It has been used as a matrix to improve the lipophilic property and targeted delivery of bioactive compounds $[5,6]$. It is expected that diosmetin combined with lecithin might result in the improvement of its lipophilic property. In this study, the complex of diosmetin with lecithin was prepared for the first time, and the physicochemical properties of the complex investigated.

\section{EXPERIMENTAL}

\section{Materials}

Diosmetin (98\%) was obtained from Shaanxi Huike Botanical Development Co, Ltd, (Xi'an, China). Lecithin from soya bean was a product of 
Sangon (Shanghai, China). Other chemical were of analytical grade.

\section{Preparation of diosmetin-lecithin complex}

Diosmetin $(100 \mathrm{mg})$ and lecithin $(200 \mathrm{mg})$ were dissolved in $100 \mathrm{ml}$ of tetrahydrofuran and stirred for $2 \mathrm{~h}$. After tetrahydrofuran was removed under vacuum, the residue was collected and pulverized. The resultant light yellow powder was collected as diosmetin-lecithin complex.

\section{Preparation of physical mixture of diosmetin and lecithin}

Diosmetin $(200 \mathrm{mg})$ and lecithin $(400 \mathrm{mg})$ were mixed and stirred in a small beaker at room temperature. The product obtained was collected as the physical mixture of diosmetin and lecithin.

\section{Ultraviolet-visible (UV) and infrared spectroscopy (IR)}

UV spectra were recorded for lecithin, diosmetin, their physical mixture and complex using a model TU1810 scanning UV spectrophotometer (Beijing Purkinje General Instrument Co., Ltd., Beijing, China). Each sample was dissolved in methanol. The absorbance of each solution was scanned in the wavelength range of $220-500 \mathrm{~nm}$ to obtain the UV spectra. IR spectra of lecithin, diosmetin, their physical mixture and complex were collected between 4000 and $400 \mathrm{~cm}^{-1}$ on a TENSOR 27 infrared spectrophotometer (Bruker, Germany) with 256 scans at a resolution of $4 \mathrm{~cm}^{-}$ ${ }^{1}$ by the $\mathrm{KBr}$ method. The data were recorded and processed by the OPUS software (Bruker, Germany) supplied with the instrument.

\section{Scanning electron microscopy (SEM)}

Analysis with scanning electron micrographs (SEM) was performed with a Quanta 200 environmental scanning electron microscope (FEI, USA). The samples were evenly distributed on SEM specimen stubs with double adhesive tape. The micrographs were obtained with an accelerating potential of $10 \mathrm{kV}$ under low vacuum.

\section{Differential scanning calorimetry (DSC)}

The samples sealed in the aluminum crimp cell were heated at the speed of $10^{\circ} \mathrm{C} / \mathrm{min}$ from 30 to $300{ }^{\circ} \mathrm{C}$ in an atmosphere of nitrogen (50 $\mathrm{ml} / \mathrm{min}$ using a DSC equipment (Q200, TA, USA). The data were recorded and processed by Universal Analysis 2000 software (TA, USA).

\section{X-ray diffractometry (XRD)}

Monochromatic $\mathrm{Cu} \mathrm{Ka}$ radiation (wavelength = $1.54056 \mathrm{~A}^{\circ}$ ) was produced by a D8 Advance Xray diffractometer (Bruker, Germany). The powdery samples were packed tightly in a rectangular aluminum cell prior to exposure to the X-ray beam. The scanning regions of the diffraction angle, $2 \theta$, were $5-70^{\circ}$ and radiation was detected with a proportional detector.

\section{RESULTS}

\section{UV and IR characteristics}

The UV spectra of lecithin, diosmetin, their physical mixture and the complex are shown in Figure 1. There was no difference between the physical mixture and the complex. The characteristic absorption peaks of diosmetin were still present at 253, 269, $344 \mathrm{~nm}$. The infrared spectra of lecithin, diosmetin, their physical mixture and the complex are shown in Figure 2. There was no significant difference between the physical mixture and the complex. The spectra of the physical mixture and the complex showed an additive effect of diosmetin and lecithin. In IR analysis, no new peaks were observed in the mixture and complex.

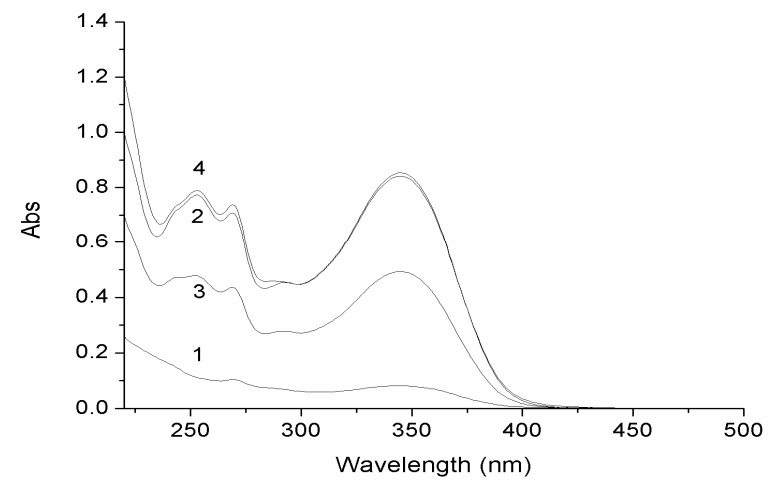

Figure 1: UV spectra of lecithin (1), diosmetin (2), their physical mixture (3) and complex (4)

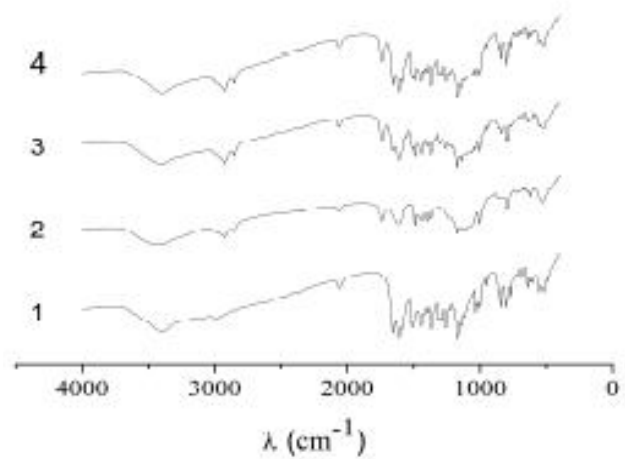

Figure 2: IR spectra of lecithin (1), diosmetin (2), their physical mixture (3) and complex (4) 


\section{SEM analysis}

The surface morphology of the complex as examined by SEM is shown in Figure 3. It could be found that diosmetin did not present in the appearance of crystal but dispersed in lecithin.
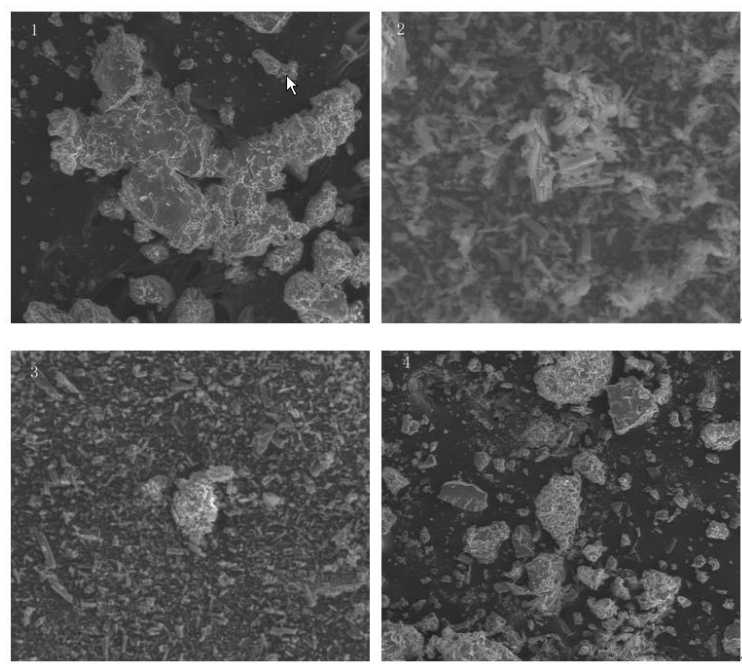

Figure 3: Scanning electron micrographs of lecithin (1), diosmetin (2), their physical mixture (3) and complex (4)

\section{Thermal characteristics}

Figure 4 shows the DSC thermograms of lecithin, diosmetin, their physical mixture and the complex. Diosmetin thermogram showed an endothermal peak with onset temperature at about $256{ }^{\circ} \mathrm{C}$, which was attributed to the melting of diosmetin. The thermogram of the physical mixture mainly showed the effect of diosmetin and lecithin. But the thermogram of the complex mainly showed the effect of lecithin, in which the characteristic endothermal peaks of diosmetin disappeared.

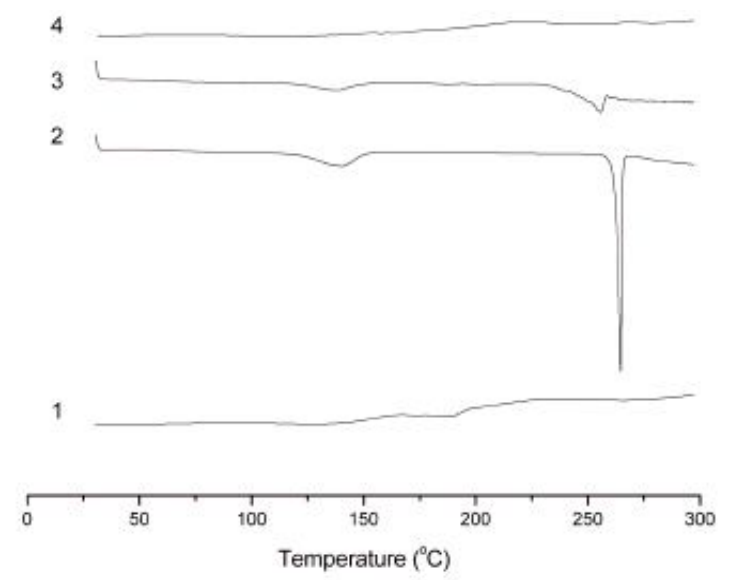

Figure 4: DSC curves of lecithin (1), diosmetin (2), their physical mixture (3) and complex (4)
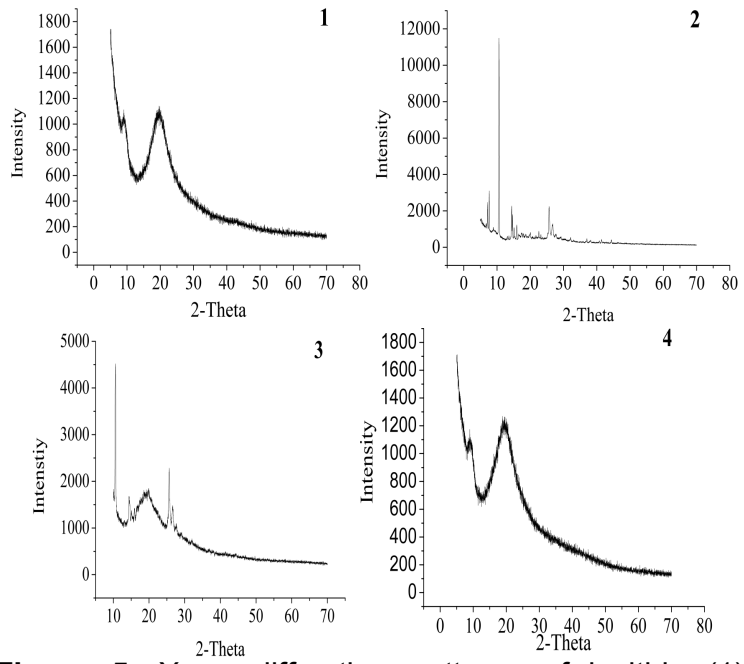

Figure 5: X-ray diffraction patterns of lecithin (1), diosmetin (2), their physical mixture (3) and complex (4)

\section{XRD analysis}

The powder X-ray diffraction patterns of diosmetin, lecithin, their physical mixture and the complex are shown in Figure 5. The powder diffraction pattern of diosmetin displayed sharp crystalline peaks, which is the characteristic of a crystalline organic molecule [7]. In contrast, lecithin showed amorphous property lacking crystalline peaks. Compared with that of the physical mixture, the crystalline peaks disappeared in the complex. This suggests that diosmetin in the lecithin matrix was molecularly dispersed. However, some crystalline drug signals were still detectable in the physical mixtures of lecithin and diosmetin.

\section{DISCUSSION}

Diosmetin-lecithin complex has been prepared using different ratios of lecithin to diosmetin, such as $1 / 10,1 / 2,1 / 1$, and $2 / 1$. The results showed that when the ratio of lecithin to diosmetin was lower than 1 , the stability of the complexes was low. In order to get optimal combinations using the lowest acceptable quantity of diosmetin, the quantity ratio of lecithin to diosmetin in the complex was determined as 2 . The obtained complex was used for the following structural analysis. The complex obtained was easy to dissolve in oil, which means that the lipophilic property of diosmetin was significantly improved by complexing with lecithin. UV and IR data indicate that there was no difference between the physical mixture and the complex, all the maximum absorption peaks of the samples were all at 253, 269, $345 \mathrm{~nm}$, which suggests that weak physical interactions between diosmetin and lecithin were involved in the formation of the complex. SEM indicates that lecithin appeared as 
amorphous while diosmetin existed in rectangular crystals. In the scanning electron micrograph of the physical mixture of the two powders, both the characteristic crystals of diosmetin and the amorphous spheres of lecithin were found. In contrast, the complex appeared as irregular shapes in which the original morphology of diosmetin disappeared. These images further demonstrate that when the powders of diosmetin and lecithin were simply mixed together, there was no close association between them and they continued to exist in their original individual forms, whereas when the complex was prepared, they formed a close association, in which diosmetin no longer existed in the crystal state.

The thermogram of the physical mixture of diosmetin and lecithin exhibited combined characteristics of the curves of diosmetin and lecithin, indicating that no close association formed between the two samples when the two powders were simply mixed together. In contrast, the thermogram of the complex exhibited mainly the features of lecithin thermogram while the characteristic endothermic peaks of diosmetin disappeared. According to a previous study [8], it was stated that diosmetin completely dispersed in lecithin and that some interaction occurred, possibly a combination of hydrogen bonds or van der Waals force.

Powder x-ray diffractometry is a useful method for the detection of compound complexation in powder or microcrystalline states. The diffraction pattern of the complex is supposed to be clearly distinct from that of the superposition of each of the components [9]. The formation of the complex between lecithin and diosmetin means that the latter might have lost its crystalline nature and, consequently, the diffraction pattern of the complex would not be a simple superposition of those of the two components [10]. The diffractogram of lecithin showed one broad peaks, consistent with its amorphous character, whereas numerous sharp, intense peaks appeared in the diffractogram of diosmetin, confirming its crystalline nature. The diffractogram of the physical mixture of the powder of diosmetin and lecithin showed essentially a superposition of the patterns of the two compounds, confirming that no association was formed between them and both retained their original physical characteristics. In contrast, the diffractogram of the complex was virtually the same as that of the amorphous lecithin and exhibited none of the characteristic peaks of diosmetin. According to the previous report [4], this suggested that diosmetin in the lecithin matrix was molecularly dispersed.

\section{CONCLUSION}

By forming a complex with lecithin, the lipophilic solubility of diosmetin is significantly enhanced. The physicochemical analysis shows that the complex was formed via non-covalent-bonds with diosmetin in the complex molecularly dispersed in lecithin matrix, and thus a new compound was not formed. The complex obtained can be used in medicine and functional food.

\section{ACKNOWLEDGEMENT}

The financial support provided by the National Science Foundation of China (21166024) is greatly appreciated

\section{REFERENCES}

1. Androutsopoulos VP, Spandidos DA. The flavonoids diosmetin and luteolin exert synergistic cytostatic effects in human hepatoma HepG2 cells via CYP1A-catalyzed metabolism, activation of JNK and ERK and P53/P21 up-regulation. J Nutr Biochem 2013; 24: 496-504

2. Chen Z, Kong S, Song F, Li L, Jiang H. Pharmacokinetic study of luteolin, apigenin, chrysoeriol and diosmetin after oral administration of Flos Chrysanthemi extract in rats. Fitoterapia 2012; 83: 1616-1622.

3. Androutsopoulos V, Wilsher N, Arroo RRJ, Potter G. Bioactivation of the phytoestrogen diosmetin by CYP1 cytochromes P450. Cancer Letters 2009; 274: 54-60.

4. Liu B, Du J, Zeng J, Chen C, Niu S. Characterization and antioxidant activity of dihydromyricetin-lecithin complex. Eur Food Res Tech 2009; 230:325-331

5. Maiti K, Mukherjee K, Gantait A, Saha BP, Mukherjee PK. Curcumin-phospholipid complex: Preparation, therapeutic evaluation and pharmacokinetic study in rats. Int J Pharm 2007; 330: 155-163.

6. Liu A, Lou H, Zhao L, Fan P. Validated LC/MS/MS assay for curcumin and tetrahydrocurcumin in rat plasma and application to pharmacokinetic study of phospholipid complex of curcumin. J Pharm Biomed Anal 2006; 40: 720-727.

7. Wang J, Cao Y, Sun B, Wang C. Characterisation of inclusion complex of trans-ferulic acid and hydroxypropyl- $\beta$-cyclodextrin. Food Chem 2011; 124 : 1069-1075.

8. Lasonder E, Weringa WD. An NMR and DSC study of the interaction of phospholipids vesicles with some antiinflammatory agents. J Colloid Interface Sci 1990; 139: 469-478.

9. Veiga F, Teixeira-Dias JJC, Kedzierewicz F, Sousa A, Maincent $P$. Inclusion complexation of tolbutamide with $\beta$-cyclodextrin and hydroxypropyl- $\beta$-cyclodextrin. Int J Pharm. 1996; 129, 63-71.

10. Liu B, Zhao J, Liu Y, Zhu X, Zeng J. Physicochemical properties of the inclusion complex of puerarin and glucosyl- $\beta$-cyclodextrin. J Agric Food Chem 2013; 61: 473-473. 